\title{
DISEÑO DE UN PROTOCOLO DE EVALUACIÓN FORMATIVA PARA EL PROGRAMA INTEGRAL DE DEPORTE ESCOLAR DEL MUNICIPIO DE SEGOVIA (PIDEMSG)
}

Design of a formative assessment protocol for the monitors of the Segovia Municipality Sports Program

Projeto de um protocolo de avaliação formativa para os monitores do Programa Integrante de Esporte Escolar de Segovia

Félix Enrique Lobo de Diego

Universidad de Valladolid, España. Teléfono: +34 686626521. Correo electrónico: flobodediego@gmail.com

\section{Resumen}

Habiendo detectado ciertas carencias en la evaluación de los aprendizajes en el PIDEMSG, se ha diseñado un protocolo de evaluación formativa, empleando fichas de observación y feedback continuos. Un estudio piloto ha comprobado la validez del protocolo mediante su aplicación con nueve monitores de Educación Infantil, Primaria y Secundaria, y un observador externo. Los instrumentos resultantes son útiles y convenientes para mejorar el aprendizaje de los escolares y ayudar a mejorar la práctica docente de los monitores. Asimismo, ha quedado patente su utilidad para complementar la retroalimentación positiva y continua habitual en la iniciación deportiva basada en modelos comprensivos.

Palabras clave: Deporte escolar; evaluación formativa; PIDEMSG

\begin{abstract}
Having detected certain lacks in the evaluation of the learning in the PIDEMSG, there has been designed a protocol of formative assessment, using cards of observation and continuous feedback. A pilot study has verified the validity of the protocol by means of if application for nine monitors of Kindergarten, Primary and Secondary Education, and an external observer. The resultant instruments are useful and suitable to improve the learning of the students and help to improve the educational practice of the monitors.

Diseño de un protocolo de evaluación formativa para los monitores del Programa Integral de Deporte Escolar del Municipio de Segovia


Likewise, its usefulness has remained clear to complement the positive and constant feedback in the sports initiation based on comprehensive models.

Keywords: School sport; formative assessment; PIDEMSG

\section{Resumo}

Tendo descoberto certas faltas na avaliação das aprendizagens em PIDEMSG, um protocolo de avaliação formativa foi projetado, enquanto usando registros de observação e avaliação contínua. Um piloto de estudo conferiu a validez do protocolo por meio de se aplicação para nove monitores de Educação Infantil, Primária e Secundária e um observador externo. Os instrumentos resultantes são úteis e convenientes melhorar a aprendizagem dos estudantes e ajudar melhorar a prática educacional dos monitores. Também, foi patente a utilidade dos registros de observação para completar o positivo e avaliação habitual contínua na iniciação desportiva baseado em modelos compreensivos.

Palavras-chave: Esportes da escola; avaliação formativa, PIDEMSG

\section{Introducción}

Alrededor del término evaluación encontramos cierta confusión debido a la falta de claridad que los profesionales tienen de este concepto. En este sentido, encontramos que muchas veces la evaluación es confundida con la calificación, mezclando así una evaluación tradicional (evaluación sumativa) basada en la racionalidad técnica y el currículum por objetivos en la que lo importante es el resultado; con una evaluación formativa que pone el énfasis en el aprendizaje y cuya base reside en la racionalidad práctica, orientada al progreso y proceso.

Álvarez (2005) sostiene que la evaluación tiene que estar al servicio del aprendizaje y los intereses formativos a los que debe servir, y no al servicio de la calificación o estar dirigida y controlada por el examen. La evaluación es la piedra angular del proceso de enseñanza y aprendizaje, y solamente cuando el aprendizaje esté garantizado se puede asegurar la evaluación, esto es, la buena evaluación, la que tiene carácter formativo y cuya finalidad según López-Pastor (2007) es 
[...] mejorar los procesos de enseñanza-aprendizaje que tienen lugar. [La evaluación formativa] Es todo proceso de evaluación que sirve para que el alumnado aprenda más (y/o corrija sus errores) y para que el profesorado aprenda a trabajar mejor (a perfeccionar su práctica docente). Por decirlo de otro modo, la finalidad principal no es calificar al alumno, sino disponer de información que permita saber cómo ayudar al alumnado a mejorar y aprender más, ... y que sirva a su vez para que los profesores aprendamos a hacer nuestro trabajo cada vez mejor. (p.64)

Anualmente en el PIDEMSG los monitores deportivos realizan una memoria en la que dejan constancia del proceso educativo realizado durante el curso escolar. No obstante, hasta el momento no se ha empleado ningún instrumento que permita ver cómo se evalúan los aprendizajes de los escolares en este programa de deporte escolar. Para dar respuesta a esta carencia detectada, este trabajo tiene como objetivo valorar la utilidad de los instrumentos diseñados para el protocolo de evaluación formativa.

\section{Método}

Para realizar una evaluación formativa en el PIDEMSG que contribuya a la mejora del aprendizaje y práctica docente, se ha diseñado un instrumento que sirve para los entrenamientos y encuentros.

Se trata de una ficha de observación (anexo I), en la que aparecen cuatro criterios generales que se corresponden con los objetivos que persigue el PIDEMSG y cuatro criterios abiertos a rellenar por los monitores (técnicos, de agrupación, etc.) en base al contenido tratado. Estos son valorados en una escala del 1 al 4, siendo 1 el mínimo y 4 el máximo. En la zona derecha se ha habilitado un espacio para que los monitores puedan tomar anotaciones que aclaren o especifiquen por qué ese criterio tiene la valoración dada.

La ficha es rellenada en cada sesión usando la técnica de la observación participante para obtener y recoger información. Este tipo de observación consiste “[...] en observar al mismo tiempo que se participa en las actividades propias del grupo que se está investigando.” (Bisquerra, 2009, pp. 332-333).

Una vez rellenada, las anotaciones tienen que ayudarles a dar retroalimentación. En la siguiente sesión o encuentro el monitor/a volverá a hacer uso de la ficha para ver si se siguen sus indicaciones, qué sale bien o mal, etc. volviendo a valorar los criterios, 
para posteriormente dar feedback sobre aquello que considere que hay que mejorar o reflexionar.

Se convierte en un proceso cíclico que hace hincapié en la mejora de la práctica deportiva como aparece en la figura 1. Acompañado de la reflexión y análisis del monitor/a sobre lo ocurrido en la sesión.

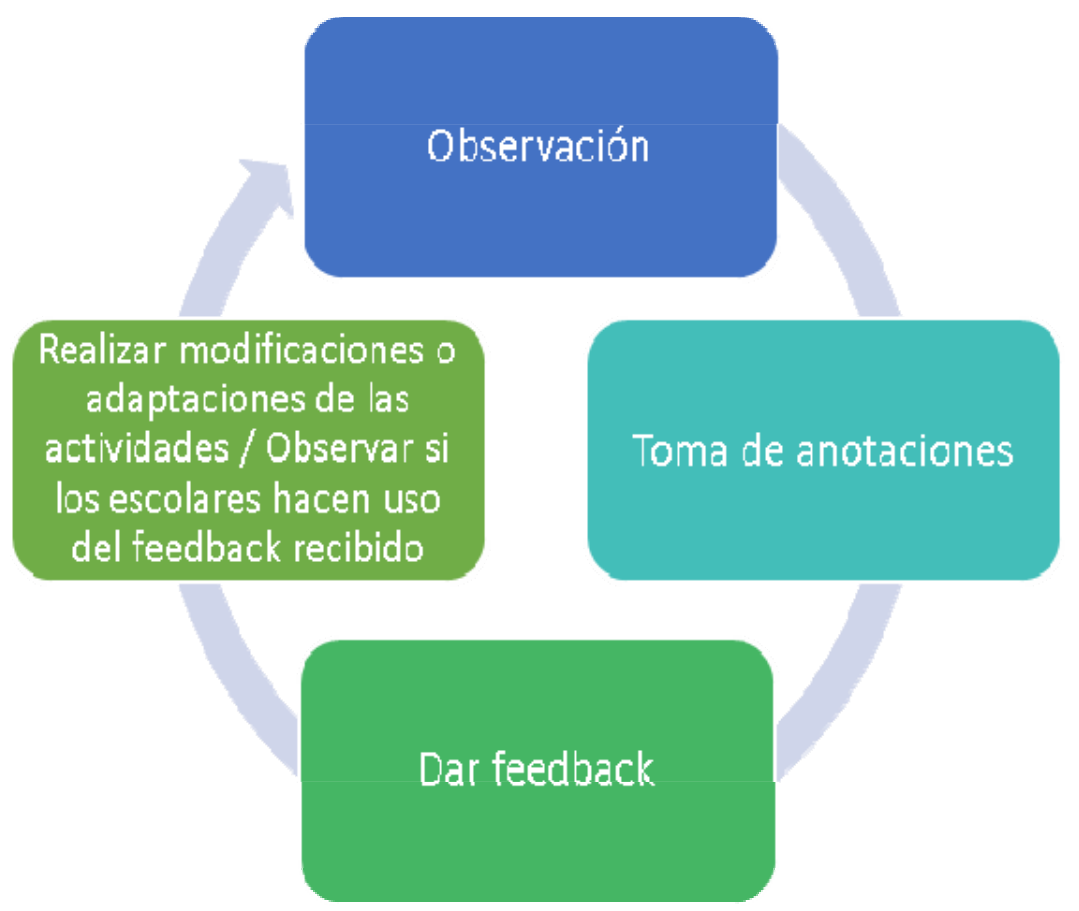

Figura 1.

Proceso que sigue el uso de las fichas de observación.

Del mismo modo, se ha diseñado una guía para monitores con menos experiencia en evaluación formativa, que ayude a rellenar la ficha con aclaraciones sobre este tipo de evaluación, cómo dar feedback, y una serie de preguntas modelo para hacer a los chicos, que permitan una retroalimentación positiva, continua y formativa.

Las fichas fueron evaluadas en un estudio piloto de dos rondas. En la primera participaron tres monitoras de Infantil y Secundaria; y en la segunda ronda seis monitores deportivos de todas las etapas educativas, y un observador externo.

Para analizar la información se ha realizado una metaevaluación por parte del observador externo, con el fin de conocer la funcionalidad de la ficha y cómo se evalúa el proceso de aprendizaje; y dos tertulias para conocer la valoración de los monitores sobre el instrumento. La información se ha analizado en base a la utilidad del Diseño de un protocolo de evaluación formativa para los monitores del Programa Integral de Deporte Escolar del Municipio de Segovia 
instrumento, frecuencia del uso de la ficha en la sesión, y frecuencia con la que se daba feedback y cómo era este.

\section{Resultados}

Tras su puesta en práctica los monitores estaban de acuerdo en que el protocolo diseñado y la ficha de observación son útiles para mejorar los aprendizajes y reflexionar sobre la propia práctica docente. Asimismo, manifestaron que las explicaciones de la guía son concisas, facilitando así el correcto desarrollo de la ficha, a la vez que ayuda a dar feedback continuo y formativo.

En cuanto al uso de la ficha, ésta fue utilizada y rellenada al final, ya que resultaba difícil rellenarla en plena sesión. Por otra parte, todos los monitores y monitoras realizaron feedback continuo en forma de pregunta para ayudar a los niños a reflexionar sobre lo que hacían, tratando en todo momento que este fuera positivo y formativo. Éste fue dado de forma individual y grupal, con el fin de que los infantes fuesen conscientes de cómo mejorar en la práctica deportiva, como se muestra en la figura 2.

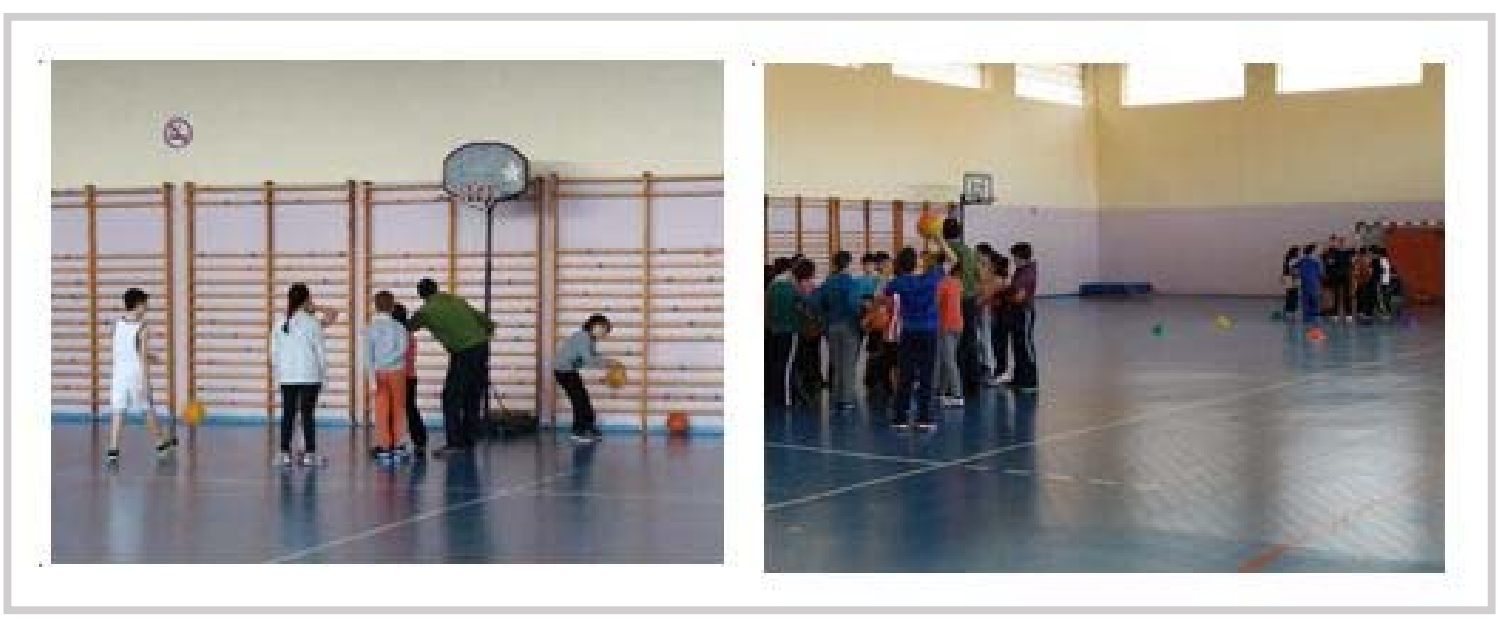

Figura 2.

Monitores dando feedback durante el estudio piloto.

\section{Conclusiones}

Tras los resultados obtenidos en la puesta en práctica de la ficha de observación, llegamos a la conclusión de que el protocolo de evaluación formativa diseñado es 
bastante útil y conveniente para realizar evaluación formativa en el PIDEMSG y mejorar los aprendizajes, cumpliendo con la finalidad para la que fue diseñado.

\section{Referencias}

Álvarez Méndez, J.M. (2005). Evaluar para conocer, examinar para excluir (segunda edición). Madrid: Ediciones Morata.

Bisquerra Alzina, R. (Coord.). (2009). Metodología de la investigación educativa (segunda edición). Madrid: La Muralla.

González-Pascual, M. (2012). Evaluación del funcionamiento del primer curso de implantación de un Programa Integral de Deporte Escolar en educación primaria en el Municipio de Segovia. (Tesis Doctoral). Valladolid: Universidad de Valladolid

López-Pastor, V. M. (2007). La evaluación en educación física y su relación con la atención a la diversidad del alumnado. Aportaciones, ventajas y posibilidades desde la evaluación formativa y compartida. Revista Kronos, 11(5), 10-15.

Pérez-Brunicardi, D. (2011). Buscando un modelo de deporte escolar para el municipio de Segovia. Un estudio a partir de las valoraciones, intereses y actitudes de sus agentes implicados. (Tesis Doctoral). Valladolid: Universidad de Valladolid. 
Anexo I. Ficha de observación diseñada

\section{Ficha de seguimiento grupal}

Fecha:

Contenido:

Grupo y centro:

Monitor/a:

\begin{tabular}{|l|l|l|}
\hline \multicolumn{1}{|c|}{ Criterio } & Valoración & Observaciones \\
\hline $\begin{array}{l}\text { 1. Participan todos los escolares y disfrutan de la } \\
\text { práctica deportiva. }\end{array}$ & & \\
\hline $\begin{array}{l}\text { 2. Valoran y aceptan las normas como parte del } \\
\text { juego o deporte. }\end{array}$ & & \\
\hline $\begin{array}{l}\text { 3. Los escolares se animan y apoyan en las } \\
\text { actividades deportivas. }\end{array}$ & & \\
\hline $\begin{array}{l}\text { 4. Aceptan las decisiones reglamentarias como } \\
\text { algo propio del juego o deporte y desde la } \\
\text { imparcialidad del árbitro (si procede). }\end{array}$ & & \\
\hline & & \\
\hline 6. ................................................................................ & & \\
\hline 7. ............................................................................... & & \\
\hline 8. .................................................................................... & & \\
\hline
\end{tabular}

Escala numérica para valoración: 1 (nunca), 2 (a veces), 3 (bastante) y 4 (siempre).

Nota para observaciones: Anotar si se logra o no el criterio, si hay excepciones (escolares que sobresalen o no llegan); aspectos motrices, sociales, etc. 
Anexo II: Guía para la realización de la ficha de observación

\section{GUÍA PARA LA FICHA DE EVALUACIÓN FORMATIVA}

- En el Programa Integral de Deporte Escolar del Municipio de Segovia (PIDEMSG), se utiliza un modelo comprensivo que implica en sí mismo la evaluación formativa, dado que dais feedback continuo.

- Este instrumento sirve para ayudaros a realizar evaluación formativa y mejorar los aprendizajes de la práctica deportiva de los escolares.

\section{Feedback}

En muchas ocasiones la mejora de los aprendizajes viene dada por el feedback ${ }^{1}$ que los monitores realizan a los escolares. A continuación, se muestra una serie de orientaciones para dar feedback.

\section{¿Cuándo dar feedback?}

- Cuando el trabajo se realiza bien, las conductas son adecuadas.

- Cuando la probabilidad de mejora de un escolar es evidente.

- Cuando observamos que un escolar está teniendo problemas de aprendizaje, o cuando la conducta de la persona tiene un impacto negativo en la actividad.

- Al finalizar la sesión.

\section{¿Cómo puede ser el feedback?}

- Concurrente: El feedback se realiza en la ejecución al mismo tiempo que el escolar modifica dicha ejecución.

- Inmediato: El feedback se realiza a los 6-20 segundos de la ejecución, conducta, etc.

- Retardado. El feedback se realiza cuando se reúne al grupo o al final de la sesión.

\footnotetext{
${ }^{1}$ El feedback debería ser siempre positivo y evitar en la medida de lo posible que sea negativo.

Diseño de un protocolo de evaluación formativa para los monitores del Programa Integral de Deporte 
- Individual: Se da a un escolar que tiene problemas de aprendizaje o conducta sin reunir al grupo.

- Grupal o Masivo: Se da a todo el grupo para comentar las dificultades de aprendizaje, problemas surgidos, felicitar por el buen trabajo realizado, etc.

\section{Preguntas orientativas para dar feedback}

A continuación encontraras preguntas o respuestas modelo que te ayuden a dar feedback.

- ¿ ¿Habéis participado todos en el juego/deporte? ... ¿ “X” te han pasado la pelota?...

¿Y por qué (o no) te la han pasado? (se puede preguntar a alguien que constantemente se la están pasando o a alguien que no la ha recibido ni una vez o pocas en la dinámica). / ¿Por qué no le habéis pasado la pelota a “X”?

- ¿Vosotros creéis que si “X” se mueve o está más cerca de los compañeros se le puede pasar la pelota con más facilidad?... Bien, pues ahora vamos a intentar movernos por el espacio y tratar de que todos reciban la pelota./ " $\mathrm{X}$ ” va a tratar de moverse para que así los compañeros podamos pasarle la pelota.

- ¿ ¿Y tú por qué crees que no te ha salido?

- Lo estás haciendo muy bien, pero si intentas hacerlo de esta forma, estoy seguro/a que te va a salir mejor.

- ¿Por qué no estáis dejando participar a “X”? ¿Si cumple las normas le vais a dejar participar?

\section{Cómo rellenar la ficha de observación}

- La ficha se valora de 1 a 4, siendo 1 el valor mínimo y 4 el máximo.

- Los cuatro primeros criterios corresponden a los objetivos e intenciones que se persiguen en el PIDEMSG. El resto de ellos son abiertos y deben ser completados por vosotros a partir de los criterios que tengáis establecidos para esta sesión o entrenamiento (no es necesario rellenar todos los criterios).

- En la zona de observaciones es importante indicar por qué se ha logrado o no el criterio, especificar si hay excepciones (escolares que sobresalen en ese criterio o que están por debajo), apuntar aquellos aspectos que permitan a los escolares mejorar o reflexionar sobre sus acciones en la práctica deportiva, etc. 
- Las anotaciones os tienen que ayudar a dar feedback continuo, positivo y formativo, que permita la mejora de los aprendizajes de los escolares y reflexionar sobre la propia práctica docente (qué ha salido bien y por qué, qué ha salido mal y por qué, cómo podríamos mejorar esa situación, dinámica para que se logre el criterio, etc.).

Diseño de un protocolo de evaluación formativa para los monitores del Programa Integral de Deporte 